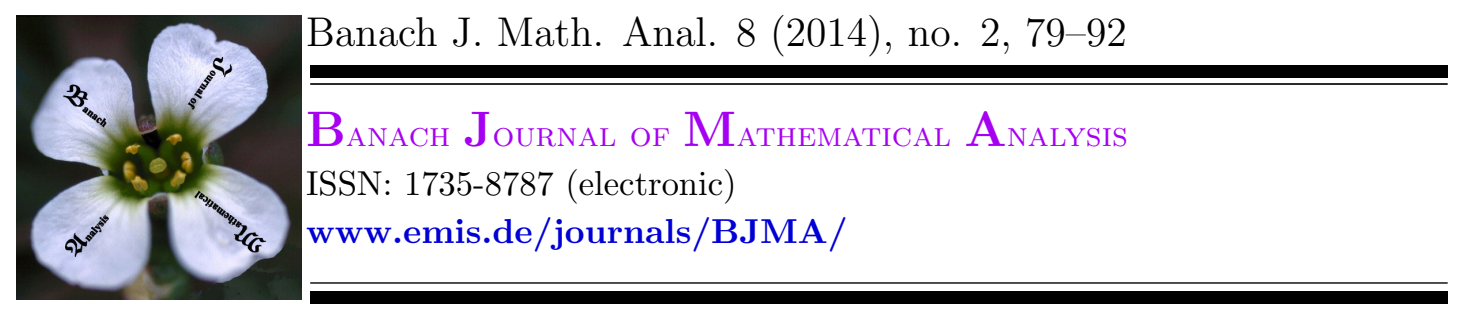

\title{
NORM-ADDITIVE IN MODULUS MAPS BETWEEN FUNCTION ALGEBRAS
}

\author{
MALIHEH HOSSEINI ${ }^{1 *}$ AND JUAN J. FONT ${ }^{2}$ \\ Communicated by R. E. Curto
}

\begin{abstract}
The main purpose of this paper is to characterize norm-additive in modulus, not necessarily linear, maps defined between function algebras (not necessarily unital or uniformly closed). In fact, for function algebras $A$ and $B$ on locally compact Hausdorff spaces $X$ and $Y$, respectively, we study surjections $T, S: A \longrightarrow B$ satisfying $\||T f|+|S g|\|_{Y}=\left|\||f|+|g|\|_{X}\right.$ for all $f, g \in A$.
\end{abstract}

\section{INTRODUCTION}

The study of preserver problems dealing with analyzing maps between Banach algebras that preserve certain spectral properties is an active research area. With regard to function algebras, most efforts have been devoted to characterize surjections preserving some given norm, spectrum and range, or certain subsets of the spectrum or of the range. For instance, the linear bijections of $C(X)$ for a compact Hausdorff space $X$, which preserve the supremum norm are determined in the famous Banach-Stone theorem $([2,27])$. The Gleason-Kahane- $\dot{Z}$ elazko theorem on the characterization of linear maps between semisimple commutative Banach algebras preserving the spectra is another well-known result in this area ([31]). Let us also recall a theorem by Kowalski and Słodkowski, in [15], which says that if a map $T: A \longrightarrow B$ between semisimple commutative Banach algebras

Date: Received: Jun. 3, 2013; Accepted: Sep. 15, 2013.

* Corresponding author.

2010 Mathematics Subject Classification. Primary 46J10; Secondary 47B38, $47 \mathrm{~B} 33$.

Key words and phrases. Norm-additive in modulus map, function algebra, Choquet boundary, uniform algebra, peaking function. 
satisfies $\sigma(T a+T b) \subseteq \sigma(a+b)$ for all $a, b \in A$, then $T$ is an algebra isomorphism, where $\sigma(\cdot)$ denotes the spectrum of the algebra element.

In [22], Molnár showed that if $X$ is a first countable compact Hausdorff space and a surjection $T: C(X) \longrightarrow C(X)$ satisfies $(T f T g)(X)=(f g)(X)$ for all $f, g \in C(X)$, then $T(1)^{-1} T$ is an algebra automorphism. Motivated by Molnár's result, some extensions to the context of uniform algebras and Banach function algebras have been given in $[8,9,10,23,24]$ and in $[18,19]$ with respect to a part of the spectrum or the range.

In [25], Rao, Tonev and Toneva studied maps $T: A \longrightarrow B$ between uniform algebras satisfying $R_{\pi}(T f+T g)=R_{\pi}(f+g)$ for all $f, g \in A$, where $R_{\pi}(f)$ is the peripheral range of $f$, i.e., the set of range values of $f$ with maximum modulus. They found sufficient conditions for such maps to be algebra isomorphisms.

In this respect, Tonev and Yates ([30]) characterized maps $T$ from a uniform algebra $A$ on a compact Hausdorff space $X$ onto a uniform algebra $B$ on a compact Hausdorff space $Y$ which are called norm-additive in modulus in [25], in the sense that $\||T f|+|T g|\|_{Y}=\||f|+|g|\|_{X}$ for all $f, g \in A$. They also gave certain conditions under which $T$ is an isometric algebra isomorphism. Moreover, in [6], Hatori, Hirasawa and Miura considered a spectral-radius additivity condition $(r(T f+T g)=r(f+g))$ for maps between unital semisimple commutative Banach algebras. From the given representation for such maps in [6], one can deduce that these maps between uniform algebras are norm-additive in modulus, but the converse is not true. For example, let $A$ be a uniform algebra on a compact Hausdorff space $X$, we can choose easily a map $\delta: A \longrightarrow\{1,-1\}$ such that the self map $T: A \longrightarrow A$ defined by $T f=\delta(f) f, f \in A$, is a normadditive in modulus surjection which is not additively spectral-radius preserving. Besides, the investigation of conditions under which maps between algebras of functions are composition or weighted composition operators has also attracted a considerable attention. For instance, the Gleason-Kahane-Żelazko theorem and the theorem by Kowalski and Słodkowski are related to this problem. Recently in [29] Tonev and Toneva gave non-linear sufficient conditions for isometries between dense subsets of uniformly closed function algebras, not necessarily unital, to be weighted composition operators and in particular, they obtained a characterization of norm-additive in modulus maps using a technique similar to the one used in the study of weak multiplicative maps ([28]), or one can see some related conclusions in [5, 13, 14, 16, 26]. Moreover, Miura characterized in [21] $\mathbb{R}$-linear isometries between function algebras and generalized some previous results. For a survey on the recent results concerning spectral preserver maps between algebras of functions, we refer the reader to [7].

The main purpose of this paper is to characterize norm-additive in modulus maps for function algebras (not necessarily unital or uniformly closed). In fact, for function algebras $A$ and $B$ on locally compact Hausdorff spaces $X$ and $Y$, respectively, we study surjections $T, S: A \longrightarrow B$ satisfying $\||T f|+|S g|\|_{Y}=$ $\||f|+|g|\|_{X}$ for all $f, g \in A$, which we call jointly norm-additive in modulus. It should be remarked that there is no linearity assumption on the operators in all these results. 


\section{PRELIMINARIES}

Let $X$ be a locally compact Hausdorff space. By $C_{0}(X)$ we mean the classical Banach algebra of all continuous complex-valued functions on $X$ vanishing at infinity, equipped with the supremum norm on $X$ denoted by $\|\cdot\|_{X}$. A subalgebra $A$ of $C_{0}(X)$ is called a function algebra on $X$ if $A$ separates strongly the points of $X$ in the sense that, given $x, z \in X$ with $x \neq z$, there is an $f \in A$ with $f(x) \neq f(z)$ and for each $x \in X$, there exists $f \in A$ with $f(x) \neq 0$. A Banach function algebra on $X$ is a function algebra on $X$ which is a Banach algebra under a certain norm. A uniformly closed function algebra on $X$ is a function algebra on $X$ which is a closed subalgebra of $\left(C_{0}(X),\|\cdot\|_{X}\right)$. Note that, when $X$ is compact, we shall assume that all function algebras on $X$ contain the constant functions, and in this case each uniformly closed function algebra on $X$ is also called a uniform algebra on $X$.

Let $A$ be a function algebra on a locally compact Hausdorff space $X$. We denote the closure of $A$ in $\left(C_{0}(X),\|\cdot\|_{X}\right)$ by $\bar{A}$. A boundary for $A$ is a subset $E$ of $X$ such that every $f \in A$ attains its maximum modulus at some point of $E$. The unique minimal closed boundary for $A$, denoted by $\partial A$, is called the $\check{S}$ ilov boundary of $A$. The Choquet boundary $C h(A)$ of $A$ is the set of all $x \in X$ for which $\delta_{x}$, the evaluation homomorphism at $x$, is an extreme point of the unit ball of the dual space of $\left(A,\|\cdot\|_{X}\right)$. Then, clearly, $C h(A)=C h(\bar{A})$. Moreover, $C h(A)$ is a boundary for $A$ and $\overline{C h(A)}=\partial A$, by [1, Theorem 1].

For an element $f$ in a function algebra $A$ on $X$, the peripheral range of $f \in A$, which is denoted by $R_{\pi}(f)$, is defined by

$$
R_{\pi}(f):=\left\{\lambda \in f(X):|\lambda|=\|f\|_{X}\right\} .
$$

It should be noted that the concept of the peripheral range of a function was introduced by Luttman and Tonev in [19].

Let $A$ be a function algebra on a locally compact Hausdorff space $X$. A function $f \in A$ is called a peaking function of $A$ if $R_{\pi}(f)=\{1\}$. We denote the set of all peaking functions of $A$ by $P_{A}$ and for a point $x \in X, P_{A}(x):=\left\{f \in P_{A}\right.$ : $f(x)=1\}$. We also call a subset $K$ of $X$ a peak set for $A$ if there exists a peaking function $f \in A$ such that $K:=\{x \in X: f(x)=1\}$. An intersection of peak sets is said to be a $p$-set. A point $x \in X$ is a strong boundary point for $A$ if for every neighborhood $V$ of $x$, there exists a function $f \in A$ such that $\|f\|_{X}=1=f(x)$ and $|f|<1$ on $X \backslash V$. It is well known that if $A$ is a uniformly closed function algebra, then $C h(A)$ is, indeed, the set of all strong boundary points for $A$ (see [17, Theorem 4.22] for compact case and [24, Theorem 2.1] for general case). But this result is not true for general Banach function algebras (although the Choquet boundary always contains all strong boundary points). For instance, the example given in [3] shows that there exists a Banach function algebra $A$ on a compact metric space $X$ with a peak set which contains no strong boundary point. Hence, since each peak set for $A$ intersects $C h(A)$, then there is a point in $C h(A)$ which is not a strong boundary point.

For an arbitrary function $f \in A$, we denote the maximum modulus set of $f$ by $M_{f}:=\left\{t \in X:|f(t)|=\|f\|_{X}\right\}$. 
An additive version of Bishop's lemma (the additive Bishop's lemma) has been proven in [25]. Here, in the proofs of our results, we apply the following analogue generalization of the additive Bishop's lemma to the context of uniformly closed function algebras:

Lemma 2.1. [30, Lemma 1] Assume that $A$ is a uniformly closed function algebra on a locally compact Hausdorff space $X$ and $f \in A$. Let $x_{0} \in C h(A)$ and arbitrary $r>1$ (or $r \geq 1$ if $f\left(x_{0}\right) \neq 0$ ), then there exists a function $h \in r\|f\|_{X} P_{A}\left(x_{0}\right)$ such that

$$
|f(x)|+|h(x)|<\left|f\left(x_{0}\right)\right|+\left|h\left(x_{0}\right)\right|
$$

for every $x \notin M_{h}$ and $|f(x)|+|h(x)|=\left|f\left(x_{0}\right)\right|+\left|h\left(x_{0}\right)\right|$ for all $x \in M_{h}$. Consequently, ||$|f|+|h| \|_{X}=\left|f\left(x_{0}\right)\right|+\left|h\left(x_{0}\right)\right|$.

Note that the preceding lemma is stated in [30] for the case where $X$ is a compact Hausdorff space $X$, but the same proof, with obvious modifications, can be also adopted directly for the locally compact case.

At the end of this section we state the following lemma which is used on several occasions in the next section:

Lemma 2.2. [1, Lemma 3] Let $X$ be a locally compact Hausdorff space, $A$ be a subalgebra of $C_{0}(X)$ and $x \in \bigcup_{f \in A} M_{f}$. If $U$ is an open subset of $X$ containing $\bigcap_{x \in M_{f}} M_{f}$, then there exists $g \in A$ such that $\|g\|_{X}=1=g(x)$ and $|g(z)|<1$ for all $z \in X \backslash U$.

\section{The RESUlts}

In the sequel, we shall assume that $A$ and $B$ are function algebras on locally compact Hausdorff spaces $X$ and $Y$, respectively. A map $T: A \longrightarrow B$ is called norm-additive in modulus $([25])$ if

$$
\||T f|+|T g|\|_{Y}=\||f|+|g|\|_{X}
$$

holds for every pair $f, g \in A$. Here we introduce a related concept:

Definition 3.1. Two maps $T, S: A \longrightarrow B$ are jointly norm-additive in modulus if

$$
\||T f|+|S g|\|_{Y}=\||f|+|g|\|_{X} \quad(f, g \in A) .
$$

Using a similar technique to [10], we introduce appropriate subsets $\tilde{X}$ and $\tilde{Y}$ of $X$ and $Y$, respectively, with some equivalence relations on them (we include all statements and details for the sake of completeness) and show that surjective jointly norm-additive in modulus maps $T, S: A \longrightarrow B$ induce a homeomorphism between the produced quotient spaces. In particular, if all points in $C h(A)$ and $C h(B)$ are strong boundary points, then $\widetilde{X}=C h(A), \widetilde{Y}=C h(B)$ and each equivalence class consists of just one element. So, in such cases, a homeomorphism is induced between $C h(A)$ and $C h(B)$.

For an element $x \in \bigcup_{f \in A} M_{f}$, set $\mathcal{I}_{x}:=\bigcap_{f \in F_{x}} M_{f}=\bigcap_{x \in M_{f}} M_{f}$, where $F_{x}:=\{f \in$ $\left.A:|f(x)|=1=\|f\|_{X}\right\}$. 
Let $\mathcal{J}:=\left\{\mathcal{I}_{x}: x \in \bigcup_{f \in A} M_{f}\right\}$. Now we define the equivalence relation $\sim$ on $\widetilde{X}:=\left\{x \in \bigcup_{f \in A} M_{f}: \mathcal{I}_{x}\right.$ is a minimal element of $\left.(\mathcal{J}, \subseteq)\right\}$ as follows: For $x, z \in \widetilde{X}$, $x \sim z$ if and only if $F_{x}=F_{z}$ (or equivalently $\mathcal{I}_{x}=\mathcal{I}_{z}$ ). Since for each $x \in \tilde{X}$, $\mathcal{I}_{x}$ is minimal, we conclude that $[x]=\mathcal{I}_{x}$. In particular, if each point in $C h(A)$ is a strong boundary point for $A$ (this holds, for example, when $A$ is either a uniformly closed function algebra or a completely regular function algebra on $X$, in the sense that all points in $X$ are strong boundary points $([11]))$, then $\widetilde{X}=C h(A)$ and for each $x \in C h(A),[x]=\{x\}$.

We also consider, similarly, the subset $\widetilde{Y}$ of $Y$ with an analogous equivalence relation on $\tilde{Y}$.

Now we state and prove a series of lemmas to be used in the proof of the main theorem (Theorem 3.5).

Lemma 3.2. Let $A$ be a function algebra on a locally compact Hausdorff space $X$ and $E$ be a p-set for $\bar{A}$. Then $C h\left(\left.\bar{A}\right|_{E}\right)=C h(A) \cap E$.

Proof. First, let us prove that the following conditions are equivalent:

a) $x \in C h(A)$.

b) For each $y \neq x$ in $X$, there is a function $f \in \bar{A}$ with $\|f\|_{X}=1=f(x)$ and $|f(y)|<1$.

We shall first show that (a) implies (b). Take $x \in C h(A)$. Since $x \in C h(A)=$ $C h(\bar{A}), x$ is a strong boundary point for $\bar{A}$ (since $\bar{A}$ is a uniformly closed function algebra) and, so, (b) is deduced easily.

Now assume that (b) holds. Let $V$ be a neighborhood of $x$. By (b), for each $y \in X \backslash V$, there exists an $f_{y} \in \bar{A}$ with $\left\|f_{y}\right\|_{X}=1=f_{y}(x)$ and $\left|f_{y}(y)\right|<1$. Then we can choose a neighborhood $V_{y}$ of $y$ such that $\left|f_{y}\right|<1$ on $V_{y}$ and $X_{\infty} \backslash V \subseteq$ $\bigcup_{y \in X \backslash V} V_{y}$. So, by the compactness of $X_{\infty} \backslash V$, there are finitely many $y_{1}, \cdots, y_{n}$ in $X \backslash V$ with $X_{\infty} \backslash V \subseteq \bigcup_{i=1}^{n} V_{y_{i}}$. Hence the function $f:=\frac{1}{n}\left(f_{y_{1}}+\cdots+f_{y_{n}}\right)$ belongs to $\bar{A},\|f\|_{X}=1=f(x)$ and $|f|<1$ on $X \backslash V$. Therefore, $x \in C h(\bar{A})=C h(A)$ and (a) is proved.

We now turn to the proof of the equation $C h\left(\left.\bar{A}\right|_{E}\right)=C h(A) \cap E$. By the above conclusion, clearly, $C h(A) \cap E \subseteq C h\left(\left.\bar{A}\right|_{E}\right)$. For the reverse inclusion, let $x \in C h\left(\left.\bar{A}\right|_{E}\right)$. Since it is obvious that $x \in E$, it suffices to check that $x \in C h(A)$. Let us suppose that $y \in X \backslash\{x\}$.

- If $y \in E$, then, since $x \in C h\left(\left.\bar{A}\right|_{E}\right)$, there is a function $\left.f \in \bar{A}\right|_{E}$ with $\|f\|_{E}=1=f(x)$ and $|f(y)|<1$. Since $E$ is a $p$-set for $\bar{A}$, there is a function $\tilde{f} \in \bar{A}$ such that $\left.\tilde{f}\right|_{E}=f$ and $\|f\|_{E}=\|\tilde{f}\|_{X}$ by $[17$, Theorem 7.4]. Hence $\tilde{f} \in \bar{A}$ with $|\tilde{f}(y)|<1=\|\tilde{f}\|_{X}=\tilde{f}(x)$.

- If $y \in X \backslash E$, take an open set $V$ containing $E$ disjoint from $y$. Since $E$ is a $p$-set, by [17, Theorem 7.1], there exists a function $f \in \bar{A}$ with $\|f\|_{X}=1$, $f=1$ on $E$ and $|f|<1$ on $X \backslash V$; in particular, $|f(y)|<1$. 
Therefore, again by the above conclusion, $x \in C h(A)$.

In the following lemma, we show that two surjective jointly norm-additive in modulus maps $T$ and $S$ are monotone increasing in modulus (see [30]). Namely:

Lemma 3.3. Let $T, S: A \longrightarrow B$ be two surjective jointly norm-additive in modulus maps. For $f, g \in A,|f| \leq|g|$ on $C h(A)$ if and only if $|T f| \leq|T g|$ (respectively, $|S f| \leq|S g|$ ) on $C h(B)$.

Proof. Let $f, g \in A$ such that $|f| \leq|g|$ on $C h(A)$. Contrary to what we claim, assume that there is a member $y_{0} \in C h(B)$ such that $\left|T f\left(y_{0}\right)\right|>\left|T g\left(y_{0}\right)\right|$. If we denote $\gamma:=\frac{1}{2}\left(\left|T f\left(y_{0}\right)\right|+\left|T g\left(y_{0}\right)\right|\right)$, then $\left|T g\left(y_{0}\right)\right|<\gamma<\left|T f\left(y_{0}\right)\right|$. Let $V$ be a neighborhood of $y_{0}$ such that $|T g|<\gamma$ on $V$. Choose a peaking function $k \in \bar{B}$ with $k\left(y_{0}\right)=1, M_{k} \subseteq V$ and $|k|<\frac{\gamma}{t}$ on $Y \backslash V$, where $t=1+\|T f\|_{Y}+\|T g\|_{Y}$. Thus, it can be easily checked that $\||T g|+t|k|\|_{Y}<\gamma+t$ and $\left|T f\left(y_{0}\right)\right|+t\left|k\left(y_{0}\right)\right|>$ $\gamma+t$. Then

$$
\||T g|+t|k|\|_{Y}<\||| T f|+t| k \mid\|_{Y}
$$

On the other hand, let $\left\{k_{n}\right\}$ be a sequence in $B$ with $\lim _{n \rightarrow \infty} k_{n}=k$. By the surjectivity of $S$ and for each $n \in \mathbb{N}$, we can choose a function $h_{n} \in A$ such that $S h_{n}=t k_{n}$. Hence, since $T$ and $S$ are jointly norm-additive in modulus maps, we have for each $n \in \mathbb{N}$,

$$
\begin{aligned}
\left\||T g|+t\left|k_{n}\right|\right\|_{Y} & =\left\||g|+\left|h_{n}\right|\right\|_{X} \\
& \geq\left\||f|+\left|h_{n}\right|\right\|_{X} \\
& =\left\||T f|+t\left|k_{n}\right|\right\|_{Y} .
\end{aligned}
$$

Letting $n \rightarrow \infty$, we obtain $\||T g|+t|k|\|_{Y} \geq\||T f|+t|k|\|_{Y}$, which contradicts (1), thus proving that $|T f| \leq|T g|$ on $C h(B)$.

Similarly, the converse can be deduced.

Furthermore, since the conditions are symmetric for $T$ and $S$, a similar argument shows that $|f| \leq|g|$ on $C h(A)$ if and only if $|S f| \leq|S g|$ on $C h(B)$.

Lemma 3.4. Let $T, S: A \longrightarrow B$ be two surjective jointly norm-additive in modulus maps and let $x \in \widetilde{X}$. There exists a point $y \in \widetilde{Y}$ such that $\bigcap_{f \in F_{x}} M_{T f}=$ $[y]=\bigcap_{f \in F_{x}} M_{S f}$.

Proof. First note that, clearly, $T$ is a norm-preserving map, i.e., $\|T f\|_{Y}=\|f\|_{X}$ for all $f \in A$.

Let us now check that $\left\{M_{T f}: f \in F_{x}\right\}$ has the finite intersection property. For this purpose, let $f_{1}, \cdots, f_{n} \in F_{x}$. Then $f=f_{1} \cdots f_{n}$ belongs to $F_{x}$. Clearly, for each $i=1, \cdots, n,|f| \leq\left|f_{i}\right|$, thus by Lemma 3.3, $|T f| \leq\left|T f_{i}\right|$ on $C h(B)$. There is a point $y \in C h(B)$ such that $|T f(y)|=1$ because $\|T f\|_{Y}=\|f\|_{X}=1$. Then, since for $i=1, \cdots, n$, we know that $\left\|T f_{i}\right\|_{Y}=\left\|f_{i}\right\|_{X}=1$, the previous inequality yields $\left|T f_{i}(y)\right|=1$. Hence $y \in \bigcap_{i=1}^{n} M_{T f_{i}}$. Then $\bigcap_{f \in F_{x}} M_{T f} \neq \emptyset$. Moreover, since every chain in the family $\mathcal{F}=\left\{I_{z}: z \in \bigcap_{f \in F_{x}} M_{T f}\right\}$ has a lower bound, then, by 
Zorn's lemma, $\mathcal{F}$ contains a minimal element, say $I_{y}$, which is, in fact, a minimal element of $\left\{I_{z}: z \in \bigcup_{g \in B} M_{g}\right\}$. Thus $y \in \tilde{Y}$ and therefore, $[y]=\mathcal{I}_{y} \subseteq \bigcap_{f \in F_{x}} M_{T f}$.

Now we prove that $\bigcap_{f \in F_{x}} M_{T f}=[y]$. Suppose not; then there exists a member $z \in \bigcap_{f \in F_{x}} M_{T f} \backslash[y]$. Choose a neighborhood $W$ of $[y]$ in $Y$ which does not contain z. By Lemma 2.2, we can find a function $g \in F_{y}$ such that $|g|<1$ on $Y \backslash W$; in particular, $|g(z)|<1$. The surjectivity of $S$ implies that $g=S f$ for some $f \in A$.

We claim that $f \in F_{x}$. If $f \notin F_{x}$ then $|f(\xi)|<1$ for every $\xi \in[x]$. Hence there exists a neighborhood $V$ of $[x]$ in $X$ such that $|f|<1$ on $V$. Choose a function $h \in F_{x}$ such that $|h|<1$ on $X \backslash V$. By hypothesis,

$$
2=\|h\|_{X}+\|f\|_{X} \geq\left\||| h|+| f\left|\left\|_{X}=\right\|\right| T h|+| S f\left|\|_{Y} \geq\right| T h(y)|+| g(y) \mid=2,\right.
$$

and consequently, $\||h|+|f|\|_{X}=2$. Then there is a member $x \in X$ such that $|h(x)|=1=|f(x)|$, which is impossible because $|f|<1$ on $V$ and $|h|<1$ on $X \backslash V$. Hence, $\bigcap_{f \in F_{x}} M_{T f}=[y]$.

By a similar argument we conclude that there is a point $y^{\prime} \in \tilde{Y}$ such that $\bigcap_{f \in F_{x}} M_{S f}=\left[y^{\prime}\right]$ and, moreover, if $g \in F_{y^{\prime}}$ and $g=T h$, then $h \in F_{x}$.

We finally claim that $y=y^{\prime}$. Indeed, if we assume that $y \neq y^{\prime}$, then, by Lemma 2.2, there exists a function $F \in F_{y}$ such that $\left|F\left(y^{\prime}\right)\right|<1$. Let $f \in A$ such that $F=S f$. According to the above, $f \in F_{x}$ and so $S f \in F_{y^{\prime}}$. Consequently, $F \in F_{y^{\prime}}$, which is a contradiction. Hence, $y=y^{\prime}$ and then $\bigcap_{f \in F_{x}} M_{T f}=[y]=\bigcap_{f \in F_{x}} M_{S f}$.

Let us denote by $\psi([x])$ the unique class $[y]$ obtained in the previous lemma. Since it depends only on $[x]$, then we can define a map $\psi: \widetilde{X} / \sim \longrightarrow \widetilde{Y} / \sim$ by $\psi([x])=[y]$ for all $x \in \widetilde{X}$, where $[y]=\bigcap_{f \in F_{x}} M_{T f}=\bigcap_{f \in F_{x}} M_{S f}$.

In the sequel, $\tau_{q}$ stands for the quotient topology on a given quotient space and by $\tau_{o}$ we mean the weakest topology under which the corresponding quotient map is open.

Theorem 3.5. (i) Suppose T, $S: A \longrightarrow B$ are surjective jointly norm-additive in modulus maps. Then there is a bijective continuous map $\psi:\left(\widetilde{X} / \sim, \tau_{o}\right) \longrightarrow$ $\left(\widetilde{Y} / \sim, \tau_{q}\right)$ such that $\psi^{-1}:\left(\widetilde{Y} / \sim, \tau_{o}\right) \longrightarrow\left(\tilde{X} / \sim, \tau_{q}\right)$ is continuous and, for each $x \in \tilde{X}$ and $f \in A$ with $\|f\|_{X} \leq 1,\|T f\|_{\psi([x])}=\|f\|_{[x]}=\|S f\|_{\psi([x])}$.

(ii) Moreover, if $T$ and $S$ are $\mathbb{R}_{+}$-homogeneous, then $\|T f\|_{\psi([x])}=\|f\|_{[x]}=$ $\|S f\|_{\psi([x])}$ for all $f \in A$ and $x \in \tilde{X}$.

Proof. (i) Let $f \in A$ with $\|f\|_{X} \leq 1$ and $x \in \tilde{X}$. We shall first show that $\|f\|_{[x]}=\|T f\|_{\psi([x])}$. Let us suppose that $\|f\|_{[x]}=0$ but $\|T f\|_{\psi([x])} \neq 0$. Then there exists a member $y_{0} \in \psi([x])$ such that $\operatorname{Tf}\left(y_{0}\right) \neq 0$. On the other hand, for $\epsilon=\min \left(\frac{\left|T f\left(y_{0}\right)\right|}{2}, 1\right)$, there exists a neighborhood $V$ of $[x]$ such that $|f|<\epsilon$ on $V$. Now, by Lemma 2.2, we can choose a function $k \in A$ such that $k \in F_{x}$ and $|k|<\epsilon$ on $X \backslash V$. Hence, according to Lemma 3.4, $S k \in F_{\psi([x])}$ and, by the 
norm-additive in modulus assumption on $T$ and $S$,

$$
\left|T f\left(y_{0}\right)\right|+1 \leq\||T f|+|S k|\|_{Y}=\||f|+|k|\|_{X} \leq \epsilon+1 .
$$

Thus $\left|T f\left(y_{0}\right)\right| \leq \epsilon$, which is a contradiction. Therefore, it follows that $\|f\|_{[x]}=$ $0=\|T f\|_{\psi([x])}$.

Let us now consider the case where $\|f\|_{[x]} \neq 0$. Since $[x]$ is compact, we can take $x^{\prime} \in[x]$ with $\|f\|_{[x]}=\left|f\left(x^{\prime}\right)\right|$.

For each $g \in F_{x^{\prime}}=F_{x}$, we claim that $M_{g}$ contains the peak set $\{t \in X: g(t)=$ $\left.g\left(x^{\prime}\right)\right\}$ for $\bar{A}$. It is apparent that $\left\{t \in X: g(t)=g\left(x^{\prime}\right)\right\} \subseteq M_{g}$. Therefore we shall show that $\left\{t \in X: g(t)=g\left(x^{\prime}\right)\right\}$ is a peak set for $\bar{A}$. Let $g\left(x^{\prime}\right)=e^{i \theta}$, where $-\pi<\theta \leq \pi$ (note that $g \in F_{x^{\prime}}$ and consequently, $\left|g\left(x^{\prime}\right)\right|=1=\|g\|_{X}$ ). Then $h=e^{-i \theta} g \in A$ and, so, the function $k=h \frac{e^{h}}{e}$ belongs to $\bar{A}$ (since $h \in A, e^{h}=$ $\sum_{k=0}^{\infty} \frac{h^{k}}{k !} \in \overline{A_{1}}$ and $\bar{A}$ is an ideal of $\left.\overline{A_{1}}\right)$. Note that Reh $\leq|h| \leq\|h\|_{X}=\|g\|_{X}=1$. Hence

$$
|k|=\left|h \frac{e^{h}}{e}\right|=|h| \frac{e^{\mathrm{Re} h}}{e} \leq \frac{e^{\mathrm{Re} h}}{e} \leq \frac{e^{1}}{e}=1 .
$$

Thus $\|k\|_{X} \leq 1$. Moreover, from the above relation and since $|h| \leq 1$, it follows that

$$
|k(t)|=1 \Leftrightarrow e^{\operatorname{Reh}(t)}=e \Leftrightarrow h(t)=1 \Leftrightarrow k(t)=1 .
$$

Therefore, $k$ is a peaking function with

$$
P(k)=\{t \in X: k(t)=1\}=\{t \in X: h(t)=1\}=\left\{t \in X: g(t)=g\left(x^{\prime}\right)\right\} .
$$

Thereby, $\left\{t \in X: g(t)=g\left(x^{\prime}\right)\right\}$ is a peak set for $\bar{A}$.

As a consequence, we have a non-empty intersection of peak sets for $\bar{A}$. Namely

$$
N_{x^{\prime}}:=\bigcap_{g \in F_{x^{\prime}}}\left\{t \in X: g(t)=g\left(x^{\prime}\right)\right\} \subseteq \bigcap_{g \in F_{x^{\prime}}} M_{g}=[x] .
$$

So, according to Lemma 3.2, $C h\left(\left.\bar{A}\right|_{N_{x^{\prime}}}\right)=C h(A) \cap N_{x^{\prime}}$. Hence there is a point $x_{0} \in C h(A) \cap N_{x^{\prime}}$ with $\left|f\left(x_{0}\right)\right|=\|f\|_{N_{x^{\prime}}}$. Then we have $\left|f\left(x^{\prime}\right)\right| \leq\|f\|_{N_{x^{\prime}}}=$ $\left|f\left(x_{0}\right)\right| \leq\|f\|_{[x]}=\left|f\left(x^{\prime}\right)\right|$, which yields $\left|f\left(x_{0}\right)\right|=\left|f\left(x^{\prime}\right)\right|=\|f\|_{[x]}$. Therefore we can also assume that $x_{0}$ is a point in $C h(A) \cap[x]$ such that $\|f\|_{[x]}=\left|f\left(x_{0}\right)\right|$. Let $V$ be an arbitrary neighborhood of $\psi([x])$ in $Y$. Our next goal is to find $y \in V$ such that $\|f\|_{[x]} \leq|T f(y)|$. Using Lemma 3.4 and Lemma 2.2, there exists a function $h^{\prime} \in F_{x}$ such that $S h^{\prime} \in F_{y}$ and $\left|S h^{\prime}\right|<\min \left(\frac{1}{2}, \frac{\left|f\left(x_{0}\right)\right|}{2}\right)$ on $Y \backslash V$. Then, by Lemma 2.1, for $x_{0} \in C h(A)$ and $r=\frac{1}{\|f\|_{X}}$, we can find a peaking function $h \in P_{\bar{A}}\left(x_{0}\right)$ with $\||f|+|h|\|_{X}=\left|f\left(x_{0}\right)\right|+1$. Let $\left\{h_{n}\right\}$ be a sequence in $A$ converging uniformly to $h$ and let $a_{n}=\left\|h_{n}\right\|_{X}^{-1}$ for all $n \in \mathbb{N}$. Thus

$$
\begin{aligned}
\|f\|_{[x]}+1 & =\left|f\left(x_{0}\right)\right|+1=\||f|+|h|\|_{X} \\
& =\left\||f|+\left|h^{\prime} h\right|\right\|_{X}=\lim _{n \rightarrow \infty}\left\||f|+\left|a_{n} h^{\prime} h_{n}\right|\right\|_{X} \\
& =\lim _{n \rightarrow \infty}\left\||T f|+\left|S\left(a_{n} h^{\prime} h_{n}\right)\right|\right\|_{Y} .
\end{aligned}
$$


Now choose $y_{n} \in C h(B)$ with $\left\||T f|+\left|S\left(a_{n} h^{\prime} h_{n}\right)\right|\right\|_{Y}=\left|T f\left(y_{n}\right)\right|+\left|S\left(a_{n} h^{\prime} h_{n}\right)\left(y_{n}\right)\right|$. Because of the compactness of $Y_{\infty}$, there is a subnet $\left\{y_{\alpha}\right\}$ of $\left\{y_{n}\right\}$ which converges to some $y \in Y_{\infty}$. Hence

$$
\|f\|_{[x]}+1=\left|f\left(x_{0}\right)\right|+1=|T f(y)|+\lim _{\alpha}\left|S\left(a_{\alpha} h^{\prime} h_{\alpha}\right)\left(y_{\alpha}\right)\right| .
$$

For each $\alpha,\left|S\left(a_{\alpha} h^{\prime} h_{\alpha}\right)\right| \leq\left|S h^{\prime}\right|$ due to Lemma 3.3. We claim that $y$ cannot be in $Y_{\infty} \backslash V$. Indeed, if $y \in Y_{\infty} \backslash V$, then $\left|S\left(a_{\alpha} h^{\prime} h_{\alpha}\right)(y)\right| \leq\left|S h^{\prime}(y)\right| \leq \min \left(\frac{1}{2}, \frac{\left|f\left(x_{0}\right)\right|}{2}\right)$. So $\|f\|_{[x]}+1=|T f(y)|+\lim _{\alpha}\left|S\left(a_{\alpha} h^{\prime} h_{\alpha}\right)\left(y_{\alpha}\right)\right| \leq 1+\left|S h^{\prime}(y)\right| \leq 1+\frac{\left|f\left(x_{0}\right)\right|}{2}=1+\frac{\|f\|_{[x]}}{2}$, which is a contradiction. Therefore, $y \in V$ and $\|f\|_{[x]}=\left|f\left(x_{0}\right)\right| \leq|T f(y)|$.

Hence, the continuity of $T f$ implies that $\|f\|_{[x]} \leq\|T f\|_{\psi([x])}$. Finally, a similar argument (for $T f$ instead of $f$ ) yields $\|T f\|_{\psi([x])} \neq 0$ and, then, the reverse inequality $\left(\|T f\|_{\psi([x])} \leq\|f\|_{[x]}\right)$ is proven in a similar way. Therefore, $\|f\|_{[x]}=$ $\|T f\|_{\psi([x])}$. Analogously, we can conclude that $\|f\|_{[x]}=\|S f\|_{\psi([x])}$.

On the other hand, as in Lemma 3.4, for each $y \in \widetilde{Y}, \bigcap_{T f \in F_{y}} M_{f}=[x]=\bigcap_{S f \in F_{y}} M_{f}$ for some $x \in \tilde{X}$ and, so, we can define a map $\varphi$ from $\tilde{Y} / \sim$ into $\tilde{X} / \sim$ such that, for each $y \in \tilde{Y}, \varphi([y])$ is the class $[x]$ given by the above relation. In like manner, we can also get $\|f\|_{\varphi([y])}=\|T f\|_{[y]}=\|S f\|_{[y]}$ for all $f \in A$ with $\|f\|_{X} \leq 1$ and $y \in \tilde{Y}$. Therefore, in particular, we have $\|f\|_{[x]}=\|f\|_{\varphi(\psi([x]))}$ and $\|T f\|_{[y]}=\|T f\|_{\psi(\varphi([y]))}$ for all $x \in \tilde{X}, y \in \tilde{Y}$ and $f \in A$ with $\|f\|_{X} \leq 1$.

Now take $x \in \widetilde{X}$ and also, for simplicity, let $\left[x^{\prime}\right]:=\varphi(\psi([x]))$. We claim that $[x]=\left[x^{\prime}\right]$. Suppose not; then there exists an open neighborhood $U$ of the compact subset $[x]=\mathcal{I}_{x}$ of $X$ with $U \cap\left[x^{\prime}\right]=\emptyset$. Hence, by Lemma 2.2, we can find a function $g \in F_{x}$ such that $|g(z)|<1$ for all $z \in X \backslash U$. Hence $\|g\|_{[x]}=1>\|g\|_{\left[x^{\prime}\right]}$, which is impossible. Thus $[x]=\varphi(\psi([x]))$ and also a similar argument shows that $[y]=\psi(\varphi([y]))$ for all $y \in \widetilde{Y}$. This implies that $\varphi$ is the inverse of $\psi$, i.e., $\psi$ is bijective.

Next, in order to get the continuity of $\psi:\left(\tilde{X} / \sim, \tau_{o}\right) \longrightarrow\left(\tilde{Y} / \sim, \tau_{q}\right)$, let $x_{0} \in \widetilde{X}$ and $y_{0} \in \widetilde{Y}$ with $\psi\left(\left[x_{0}\right]\right)=\left[y_{0}\right]$. Let $\widetilde{W}$ be a neighborhood of $\left[y_{0}\right]$ in the quotient topology on $\tilde{Y} / \sim$. Then there is a neighborhood $W_{0}$ of $y_{0}$ in $Y$ with $\pi_{2}^{-1}(\widetilde{W})=\widetilde{Y} \cap W_{0}$, where $\pi_{2}: \tilde{Y} \longrightarrow \widetilde{Y} / \sim$ is the quotient map. Clearly $\left[y_{0}\right] \subseteq W_{0}$. Hence, by Lemma 2.2, there exists a function $g \in A$ such that $T g\left(y_{0}\right)=1=\|T g\|_{Y}$ and $|T g|<1 / 2$ on $Y \backslash W_{0}$; in particular, $|T g|<1 / 2$ on $\widetilde{Y} \backslash \pi_{2}^{-1}(\widetilde{W})$. By the preceding explanations, $\|g\|_{[x]}=\|T g\|_{\psi([x])}=\|S g\|_{\psi([x])}$. Thus if we let

$$
\widetilde{V}:=\left\{[x] \in \tilde{X} / \sim:\|T g\|_{\psi([x])}>1 / 2\right\}=\left\{[x] \in \widetilde{X} / \sim:\|S g\|_{\psi([x])}>1 / 2\right\},
$$

it suffices to check that $\widetilde{V}$ is a neighborhood of $\left[x_{0}\right]$ in $\left(\widetilde{X} / \sim, \tau_{o}\right)$ and that $\psi(\widetilde{V}) \subseteq \widetilde{W}$. Since $y_{0} \in \psi\left(\left[x_{0}\right]\right)$ and $\|T g\|_{\psi\left(\left[x_{0}\right]\right)} \geq\left|(T g)\left(y_{0}\right)\right|>1 / 2$, then $\|g\|_{\left[x_{0}\right]}=$ $\|T g\|_{\psi\left(\left[x_{0}\right]\right)}>1 / 2$. Consequently, there exists $x^{\prime} \in\left[x_{0}\right]$ with $\left|g\left(x^{\prime}\right)\right|>1 / 2$. Choose a neighborhood $U$ of $x^{\prime}$ in $X$ such that $|g|>1 / 2$ on $U$. In particular, $|g(x)|>1 / 2$ for all $x \in U \cap \tilde{X}$. Then $\|T g\|_{\psi([x])}=\|g\|_{[x]}>1 / 2$, i.e., $[x] \in \widetilde{V}$. Hence 
$\widetilde{U}=\pi_{1}(U \cap \widetilde{X})$ is a neighborhood of $\left[x^{\prime}\right]=\left[x_{0}\right]$ such that $\widetilde{U} \subseteq \widetilde{V}$, where $\pi_{1}$ : $\widetilde{X} \longrightarrow \widetilde{X} / \sim$ is the quotient map. Therefore, $\widetilde{V}$ is a neighborhood of $\left[x_{0}\right]$ in $\left(\widetilde{X} / \sim, \tau_{o}\right)$. Moreover, $\psi(\widetilde{V}) \subseteq \widetilde{W}$ because if $[x] \in \widetilde{V}$, then $\|T g\|_{\psi([x])}>1 / 2$ and, so, $|T g(y)|>1 / 2$ for some $y \in \psi([x])$. Thus $y \in \widetilde{Y} \cap W_{0}=\pi_{2}^{-1}(\widetilde{W})$. Consequently, $\psi([x])=[y] \in \widetilde{W}$ and, therefore, $\psi$ is continuous.

Similarly, $\psi^{-1}:\left(\tilde{Y} / \sim, \tau_{o}\right) \longrightarrow\left(\tilde{X} / \sim, \tau_{q}\right)$ is continuous as well.

(ii) Let $f \in A$ and $x \in \widetilde{X}$. Since $T$ is $\mathbb{R}_{+}$-homogenous, by (i) it follows that

$$
\|T f\|_{\psi([x])}=\|f\|_{X} \cdot\left\|T\left(\frac{f}{\|f\|_{X}}\right)\right\|_{\psi([x])}=\|f\|_{X} \cdot\left\|\frac{f}{\|f\|_{X}}\right\|_{[x]}=\|f\|_{[x]},
$$

i.e., $\|f\|_{[x]}=\|T f\|_{\psi([x])}$. Similarly, we may conclude that $\|f\|_{[x]}=\|S f\|_{\psi([x])}$

As mentioned before, if each point in $C h(A)$ is a strong boundary point for $A$, then $\widetilde{X}=C h(A)$ and $[x]=\mathcal{I}_{x}=\{x\}$ for all $x \in C h(A)$. Furthermore, in these cases the quotient maps are identity maps and the topologies $\tau_{q}$ and $\tau_{o}$ are the same relative topology of spaces.

Note also that there are several classical function algebras with the property that the points in the Choquet boundary are strong boundary points, such as uniformly closed function algebras, algebras of Lipschitz functions $(\operatorname{Lip}(X))$, of little Lipschitz functions $(\operatorname{lip}(X))$, of the (pointed) Lipschitz functions $\left(\operatorname{Lip}_{0}(X)\right)$, of bounded variation continuous functions $(B V C(X))$, of absolutely continuous functions $(A C(X))$ and of n-times continuously differentiable functions $\left(C^{(n)}(X)\right.$, $n \in \mathbb{N} \cup\{0, \infty\})$ on appropriate compact spaces $X$. It is interesting to note that when $X$ is a perfect compact plane set which is a finite union of uniformly regular sets (see [4]), the algebra $C^{\infty}(X)$ (for example, $C^{\infty}(I)$, where $I=[0,1]$ ) is not a Banach function algebra.

Thus we can deduce the following corollaries easily:

Corollary 3.6. If all points in $C h(A)$ and $C h(B)$ are strong boundary points, $T$ and $S$ are $\mathbb{R}_{+}$-homogenous and jointly norm-additive in modulus surjections, then there exists a homeomorphism $\varphi: C h(B) \longrightarrow C h(A)$ such that for all $y \in C h(B)$ and $f \in A$,

$$
|T f(y)|=|f(\varphi(y))|=|S f(y)| .
$$

In particular, by assuming $S=T$, the above result can be applied for normadditive in modulus surjections. We recall that such maps between uniform algebras have recently been studied in [25, 30], and moreover, between dense subalgebras of uniformly closed function algebras in [29]. Corollary 3.6 implies [25, Lemma 14 and Corollary 6]. Moreover, since each dense subalgebra of a uniformly closed function algebra is a function algebra, the preceding result generalizes [30, Proposition 10] and [29, Proposition 3.1] without the injectivity assumption for $T$.

Corollary 3.7. Let $\left(X, d_{1}\right)$ and $\left(Y, d_{2}\right)$ be compact metric spaces and let $T, S$ : $\operatorname{Lip}(\mathrm{X}) \longrightarrow \operatorname{Lip}(\mathrm{Y})$ be $\mathbb{R}_{+}$-homogenous and jointly norm-additive in modulus surjections. Then there exists a bi-Lipschitz homeomorphism $\varphi: Y \longrightarrow X$ such that 
for all $y \in Y$ and $f \in \operatorname{Lip}(\mathrm{X})$,

$$
|T f(y)|=|f(\varphi(y))|=|S f(y)| .
$$

Proof. Since Lipschitz algebras are completely regular, then, by Corollary 3.6 there exists a homeomorphism $\varphi: Y \longrightarrow X$ such that $|T f(y)|=|f(\varphi(y))|$ for all $y \in Y$ and $f \in \operatorname{Lip}(\mathrm{X})$. Assume now that $\varphi$ is not a Lipschitz map. Then, by [12, Lemma 2.3], there exist sequences $\left\{y_{n}\right\}$ and $\left\{y_{n}^{\prime}\right\}$ in $Y$ converging to a point $y \in Y$ such that $y_{n} \neq y_{n}^{\prime}$ and

$$
n<\frac{d_{1}\left(\varphi\left(y_{n}\right), \varphi\left(y_{n}^{\prime}\right)\right)}{d_{2}\left(y_{n}, y_{n}^{\prime}\right)}
$$

for all $n \in \mathbb{N}$ and a function $f \in \operatorname{Lip}(\mathrm{X})$ with $f\left(\varphi\left(y_{n}\right)\right)=d_{1}\left(\varphi\left(y_{n}\right), \varphi\left(y_{n}^{\prime}\right)\right)$ and $f\left(\varphi\left(y_{n}^{\prime}\right)\right)=0$. In particular, $T f\left(y_{n}^{\prime}\right)=0$. Thus we have

$$
n<\frac{d_{1}\left(\varphi\left(y_{n}\right), \varphi\left(y_{n}^{\prime}\right)\right)}{d_{2}\left(y_{n}, y_{n}^{\prime}\right)}=\frac{\left|f\left(\varphi\left(y_{n}\right)\right)-f\left(\varphi\left(y_{n}^{\prime}\right)\right)\right|}{d_{2}\left(y_{n}, y_{n}^{\prime}\right)}=\frac{\left|T f\left(y_{n}\right)-T f\left(y_{n}^{\prime}\right)\right|}{d_{2}\left(y_{n}, y_{n}^{\prime}\right)} \leq L(T f)
$$

for all $n \in \mathbb{N}$, which is impossible since $T f \in \operatorname{Lip}(\mathrm{Y})$. Therefore, $\varphi$ is a Lipschitz map. Similarly, $\varphi^{-1}$ is a Lipschitz map on $X$, and, so, $\varphi$ is a bi-Lipschitz homeomorphism.

Remark 3.8. A similar result is valid for the pointed Lipschitz algebras. Indeed, since the pointed Lipschitz algebra $\operatorname{Lip}_{0}(\mathrm{X})$, for a pointed compact metric space $X$ with the base point $e_{X}$, is a Banach function algebra on the locally compact Hausdorff space $X \backslash\left\{e_{X}\right\}$ and every point in $X \backslash\left\{e_{X}\right\}$ is a strong boundary point, then, similar to the above proof and applying [12, Lemma 2.4], we conclude that Corollary 3.7 is also true for the pointed Lipschitz algebras.

We would like to note that if we assume $S=T$ in the previous corollaries, then the map $T$ is a composition operator in modulus which is not necessarily linear or multiplicative. For example, as pointed out before, if $A$ is a function algebra on a compact Hausdorff space $X$, then a map $\delta: A \longrightarrow\{1,-1\}$ can be chosen such that the self map $T: A \longrightarrow A$ defined by $T f:=\delta(f) f, f \in A$, is a surjective norm-additive in modulus map which is neither $\mathbb{R}$-linear nor multiplicative. In contrast, if we assume that a norm-additive in modulus surjection $T: A \longrightarrow B$ is additive, then since $T 0=0,\|T f+T g\|_{Y}=\|T(f+g)\|_{Y}=\|f+g\|_{X}$ and again because of the equation $T 0=0$, we conclude that $\|T f-T g\|_{Y}=\|f-g\|_{X}$ for all $f, g \in A$. Hence by the Mazur-Ulam theorem ([20]), $T$ is an $\mathbb{R}$-linear isometry between the uniform closures of the algebras and so according to [21, Theorem 1.1] we can obtain its representation. If, furthermore, $T(i h)=i T(h)$ for some $h \in A$ with $h \neq 0$ on $C h(A)$, hence $T$ is a weighted composition operator on the Choquet boundary of $B$. Moreover, in [29, Theorem 4.1] it has been proven that if the latter condition is replaced by $R_{\pi}(T f)=R_{\pi}(f)$ for all $f \in A$, then $T$ is an algebra isomorphism. Note that these recent results are true when $T$ is defined between dense subsets of $A$ and $B$ since $T$ is an $\mathbb{R}$-linear isometry and so it can be extended naturally (see [29]). 
Corollary 3.9. Let $m, n \in \mathbb{N} \cup\{0, \infty\}, T, S: C^{(m)}(I) \longrightarrow C^{(n)}(I)$ be additive, jointly norm-additive in modulus surjections such that $T(i h)=i T(h)$ and $S(i k)=$ $i S(k)$ for some $h, k \in C^{(m)}(I)$ with $h, k \neq 0$ on $I$. Then $m=n$ and there exists a $C^{(n)}$-diffeomorphism $\varphi: I \longrightarrow I$ such that $\frac{T f(y)}{T 1(y)}=f(\varphi(y))=\frac{S f(y)}{S 1(y)}$ for each $f \in C^{(n)}(I)$ and $y \in I$.

Proof. From the above explanations and Corollary 3.6 it follows that there is a homeomorphism $\varphi: I \longrightarrow I$ such that $\frac{T f(y)}{T 1(y)}=f(\varphi(y))=\frac{S f(y)}{S 1(y)}$ for all $y \in I$ and $f \in C^{(m)}(I)$.

We now show that $\varphi \in C^{(n)}(I)$. Let $v \in \mathbb{C} \backslash\{0\}$ and put $f \in C^{(m)}(I)$ defined by $f(x)=x v$ for all $x \in I$. Then for each $y \in I$,

$$
T f(y)=T 1(y) f(\varphi(y))=T 1(y) \varphi(y) v .
$$

Hence $\varphi=\frac{T f}{v T 1}$ and since $v \neq 0,|T 1|=1$, it follows easily that $\varphi \in C^{(n)}(I)$. Similarly, $\varphi^{-1} \in C^{(m)}(I)$.

Next, we show that $m=n$. Let $f \in C^{(m)}(I)$. Thus $g=f \circ \varphi^{-1} \in C^{(m)}(I)$. Fix $u \in \mathbb{C} \backslash\{0\}$ and put $h=g u$. Then $h \in C^{(m)}(I)$ and hence $T h \in C^{(n)}(I)$. Moreover, for each $y \in I$,

$$
T h(y)=T 1(y) g(\varphi(y)) u=T 1(y) f(y) u .
$$

Thus $f=\frac{T h}{u T 1} \in C^{(n)}(I)$ since $u$ is not zero and $|T 1|=1$. Therefore, $C^{(m)}(I) \subseteq$ $C^{(n)}(I)$ and so $m \geq n$. By symmetry, $n \geq m$ and then $m=n$.

Acknowledgement. The authors would like to thank the referee for his/her useful comments.

Research of J.J. Font was partially supported by the Spanish Ministry of Science and Education (Grant number MTM2011-23118), and by Bancaixa (Projecte P11B2011-30).

\section{REFERENCES}

1. J. Araujo and J.J. Font, On S̆ilov boundaries for subspaces of continuous functions, Topology Appl. 77 (1997), 79-85.

2. S. Banach, Théorie des Opérations Linéaires, AMS Chelsea Publishing, Warsaw, 1932.

3. H.G. Dales, Boundaries and peak points for Banach function algebras, Proc. London Math. Soc. 22 (1971), 121-136.

4. H.G. Dales and A.M. Davie, Quasianalytic Banach function algebras, J. Funct. Anal. 13 (1973), 28-50.

5. O. Hatori, K. Hino, T. Miura and H. Oka, Peripherally monomial-preserving maps between uniform algebras, Mediterr. J. Math. 6 (2009), 47-59.

6. O. Hatori, G. Hirasawa and T. Miura, Additively spectral-radius preserving surjections between unital semisimple commutative Banach algebras, Cent. Eur. J. Math. 8 (2010), $597-601$.

7. O. Hatori, S. Lambert, A. Lutman, T. Miura, T. Tonev and R. Yates, Spectral preservers in commutative Banach algebras, Contemp. Math. 547 (2011), 103-123.

8. O. Hatori, T. Miura and H. Takagi, Characterizations of isometric isomorphisms between uniform algebras via nonlinear range-preserving properties, Proc. Amer. Math. Soc. 134 (2006), 2923-2930. 
9. O. Hatori, T. Miura and H. Takagi, Unital and multiplicatively spectrum-preserving surjections between commutative Banach algebras are linear and multiplicative, J. Math. Anal. Appl. 326 (2007), 281-296.

10. M. Hosseini and F. Sady, Multiplicatively range-preserving maps between Banach function algebras, J. Math. Anal. Appl. 357 (2009), 314-322.

11. K. Jarosz and V. Pathak, Isometries and small bound isomorphisms of function spaces, Lecture Notes in Pure and Appl. Math. 136, Dekker (1992), 241-271.

12. A. Jiménez-Vargas, K. Lee, A. Luttman and M. Villegas-Vallecillos, Generalized weak peripheral multiplicativity in algebras of Lipschitz functions, Cent. Eur. J. Math. 11 (2013), 1197-1211.

13. A. Jiménez-Vargas and M. Villegas-Vallecillos, Lipschitz algebras and peripherallymultiplicative maps, Acta Math. Sin. (Engl. Ser.) 24 (2008), 1233-1242.

14. J. Johnson and T. Tonev, Spectral conditions for weighted composition operators on function algebras, Commun. Math. Appl. 3 (2012), 51-59.

15. S. Kowalski and Z. Słodkowski, A characterization of maximal ideals in commutative Banach algebras, Studia Math. 67 (1980), 215-223.

16. K. Lee and A. Luttman, Generalizations of weakly peripherally multiplicative maps between uniform algebras, J. Math. Anal. Appl. 375 (2011), 108-117.

17. G.M. Leibowitz, Lectures on Complex Function Algebras, Scott, Foresman and Company, 1970.

18. S. Lambert, A. Luttman and T. Tonev, Weakly peripherally-multiplicative mappings between uniform algebras, Contemp. Math. 435 (2007), 265-281.

19. A. Luttman and T. Tonev, Uniform algebra isomorphisms and peripheral multiplicativity, Proc. Amer. Math. Soc. 135 (2007), 3589-3598.

20. S. Mazur and S. Ulam, Sur les transformations isométriques d'espaces vectoriels normés, C. R. Acad. Sci. Paris 194 (1932), 946-948.

21. T. Miura, Real-linear isometries between function algebras, Cent. Eur. J. Math. 9 (2011), $778-788$.

22. L. Molnár, Some characterizations of the automorphisms of $B(H)$ and $C(X)$, Proc. Amer. Math. Soc. 130 (2002), 111-120.

23. N.V. Rao and A.K. Roy, Multiplicatively spectrum-preserving maps of function algebras, Proc. Amer. Math. Soc. 133 (2005), 1135-1142.

24. N.V. Rao and A.K. Roy, Multiplicatively spectrum-preserving maps of function algebras II, Proc. Edinb. Math. Soc. 48 (2005), 219-229.

25. N.V. Rao, T. Tonev and E. Toneva, Uniform algebra isomorphisms and peripheral spectra, Contemp. Math. 427 (2007), 401-416.

26. R. Shindo, Weakly-peripherally multiplicative conditions and isomorphisms between uniform algebras, Publ. Math. Debrecen 78 (2011), 675-685.

27. M.H. Stone, Applications of the theory of Boolean rings to general topology, Trans. Amer. Math. 41 (1937), 375-481.

28. T. Tonev, Weak multiplicative operators on function algebras without units, Banach Center Publications 91 (2010), 411-421.

29. T. Tonev and E. Toneva, Composition operators between subsets of function algebras, Contemp. Math. 547 (2011), 227-237.

30. T. Tonev and R. Yates, Norm-linear and norm-additive operators between uniform algebras, J. Math. Anal. Appl. 357 (2009), 45-53.

31. W. Żelazko, Banach Algebras, Elsevier, Amsterdam-London-New York, 1973.

${ }^{1}$ Department of Mathematics, K.N. Toosi University of Technology, Tehran, 16315-1618, IRAN.

E-mail address: m.hosseini@kntu.ac.ir 
2 Departamento de Matemáticas, Universitat Jaume I, Campus Riu Sec, 8029 AP, Castellón, Spain.

E-mail address: font@mat.uji.es 